\title{
Article \\ Study of Type II SPDC in Lithium Niobate for High Spectral Purity Photon Pair Generation
}

\author{
Ilhwan Kim, Donghwa Lee and Kwang Jo Lee *(D) \\ Department of Applied Physics, Institute of Natural Science, Kyung Hee University, Yongin-si 17104, Korea; \\ dlfghks383@gmail.com (I.K.); fairytale095@gmail.com (D.L.) \\ * Correspondence: kjlee88@khu.ac.kr
}

check for updates

Citation: Kim, I.; Lee, D.; Lee, K.J. Study of Type II SPDC in Lithium Niobate for High Spectral Purity Photon Pair Generation. Crystals 2021, 11, 406. https://doi.org/10.3390/ cryst11040406

Academic Editors: Gábor Corradi and László Kovács

Received: 3 March 2021

Accepted: 9 April 2021

Published: 10 April 2021

Publisher's Note: MDPI stays neutral with regard to jurisdictional claims in published maps and institutional affiliations.

Copyright: (c) 2021 by the authors. Licensee MDPI, Basel, Switzerland. This article is an open access article distributed under the terms and conditions of the Creative Commons Attribution (CC BY) license (https:// creativecommons.org/licenses/by/ $4.0 /)$.

\begin{abstract}
Recent advances of high-quality lithium niobate (LN) on insulator technology have revitalized the progress of novel chip-integrated LN-based photonic devices and accelerated application research. One of the promising technologies of interest is the generation of entangled photon pairs based on spontaneous parametric down-conversion (SPDC) in LNs. In this paper, we investigated, theoretically and numerically, Type II SPDC in two kinds of LNs-undoped and 5-mol\% MgO doped LNs. In each case, both non-poled and periodically poled crystals were considered. The technique is based on the SPDC under Type II extended phase matching, where the phase matching and the group velocity matching are simultaneously achieved between interacting photons. The proposed approach has not yet been reported for LNs. We discussed all factors required to generate photon pairs in LNs, in terms of the beam propagation direction, the spectral position of photons, and the corresponding effective nonlinearities and walk-offs. We showed that the spectral positions of the generated photon pairs fall into the mid-infrared region with high potential for free-space quantum communication, spectroscopy, and high-sensitivity metrology. The joint spectral analyses showed that photon pairs can be generated with high purities of 0.995-0.999 with proper pump filtering.
\end{abstract}

Keywords: lithium niobate; parametric down-conversion; photon-pair generation; extended phase matching

\section{Introduction}

Lithium niobate $\left(\mathrm{LiNbO}_{3}, \mathrm{LN}\right)$ has been the most preferred nonlinear optic platform for decades due to its high material quality, mature manufacturing technology, large second-order nonlinearity and electro-optic (EO) modulation efficiency [1]. In particular, the ion-cut technology for manufacturing wafer-size, low propagation loss, sub-micronthick crystalline LN thin films has made significant advances over the past decade [2,3]. This technique allows the successful integration of undoped or $\mathrm{MgO} / \mathrm{Fe} / \mathrm{Er} / \mathrm{Tm}$ doped $\mathrm{LN}$ on insulators such as $\mathrm{SiO}_{2} / \mathrm{Si}$, $\mathrm{Si}$, sapphire, and quartz [4]. This rapid development of LN-on-insulator (LNOI) technology has revitalized the progress of novel chip-integrated LN-based photonic devices and accelerated application research [5-17]. The monolithically integrated LN modulator with on-chip loss of less than $0.5 \mathrm{~dB}$ and the high modulation rate of $210 \mathrm{~Gb} / \mathrm{s}$ has been successfully demonstrated, which was followed by many promising applications including on-chip photonic integration, Kerr frequency comb or supercontinuum generation, and nonlinear-optic quasi-phase-matching (QPM) frequency conversion on an integrated platform [5-7]. Chip-based LNOI light sources including frequency combs, on-chip self-referencing systems, and a two-octave spanning supercontinuum have been demonstrated [8-10]. On-chip integration of spectrometers, modulators, resonators, frequency shifters, and periodically poled LN (PPLN) waveguides for integrated photonic circuit construction has also been reported recently [11-17].

One of the important applications of second-order nonlinearity is the generation of entangled photon pairs based on spontaneous parametric down-conversion (SPDC) in $\chi^{(2)}$ crystals [18]. In particular, two photons generated simultaneously via Type II SPDC have 
orthogonal polarizations in the basis that are distinguished by crystallographic axes. In this case, the entangled photon pairs are obtained from the spatial region where two beams (or cones) with each polarization overlap, or from the Sagnac-loop interferometer configuration $[19,20]$. In addition, the extended phase matching (EPM) is defined as when group velocity $(\mathrm{GV})$ matching is additionally achieved between photons interacting under Type II SPDC, where high-purity photon pairs can be generated in a wide bandwidth [21-25]. Several studies on the photon-pair generation based on Type II SPDC in PPLN waveguides have been reported so far, but in all cases the EPM scheme was not used or the frequencies of the generated photon pairs were not identical to each other [26-30]. The experimentally reported brightness values are $6 \times 10^{5} / \mathrm{s} / \mathrm{GHz}$ and $3 \times 10^{5} / \mathrm{s} / \mathrm{GHz}$ for non-diffused and Ti-indiffused PPLN waveguides, respectively [26,27]. The rates of photon-pair generation are $1.2 \times 10^{3}$ pairs $/ \mathrm{s} / \mathrm{mW}$ and $2.87 \times 10^{7}$ pairs $/ \mathrm{s} / \mathrm{mW}$ for a bulk PPLN and hybrid PPLN waveguide, respectively [28,29]. Experimental results for the generation of photon pairs in thin-film PPLN have only been reported recently, where Type 0 SPDC were used [31,32]. The brightness is $69 \times 10^{6} / \mathrm{s} / \mathrm{mW} / \mathrm{nm}$, and the photon-pair generation rates are 91 pairs $/(\mathrm{s}$ $\mathrm{GHz} \mathrm{Mw}$ ) for Type 0 and 44,360 pairs/(s GHz mW) for Type I, respectively [31,32]. In addition, Type II EPM approach has not yet been reported for non-poled LNs. However, in order to generate high-purity photon pairs that are indistinguishable except for the state of polarization, SPDC based on frequency-degenerate Type II EPM is required. Therefore, it is timely to investigate the usefulness of LN as another suitable candidate for the generation of frequency-degenerate, entangled photon pairs while meeting all requirements.

In this paper, we investigate, both theoretically and numerically, Type II SPDC in two kinds of LNs (i.e., undoped and 5-mol\% MgO doped LNs), which are expected to be useful as material platforms for the generation of entangled photon pairs of high spectral purity. In each case, both non-poled and PP crystals will be considered. The 5-mol\% MgO-doping prevents photorefractive damage to LN (optically induced refractive index change when the crystal is exposed to blue or green CW light) $[33,34]$. The technique is based on the SPDC under Type II EPM, where the phase matching (QPM or birefringent PM) and the GV matching are simultaneously achieved between the interacting photons in both nonpoled and PP crystals. First, we investigate the Type II EPM characteristics for non-poled and PPLN in two types of LN, respectively, in terms of the beam propagation direction, the spectral position of photon pairs, and the corresponding effective nonlinearities and beam walk-offs. We will show that the spectral positions of the generated photon pairs fall into the mid-infrared (mid-IR) region with high potential for free-space quantum communication, spectroscopy, and high-sensitivity metrology [35-38]. The joint spectral analyses showed that photon pairs can be generated with high purities of 0.995-0.999 with proper pump filtering.

\section{Materials and Theories}

LN belongs to the trigonal point group $3 \mathrm{~m}$ at room temperature, and exhibits negative uniaxial birefringence [1]. In this case, the order of refractive-index (RI) magnitudes is given by $n_{e}<n_{0}$, where $n_{e}$ and $n_{0}$ represent the RIs of the extraordinary and ordinary waves in the principal axes of the index ellipsoid $[39,40]$. Figure 1 illustrates the frequency and polarization relationships of pump, signal, and idler photons interacting via Type II SPDC. In a non-poled LN shown in Figure 1a, an input pump photon with a frequency $2 \omega$ produces a pair of photons (signal and idler) with the same frequency $\omega$ and polarization states perpendicular to each other. In this case, the pump wave vector that satisfies Type II EPM generally does not propagate along the crystallographic axis, resulting in a spatial walk-off between the interacting beams as illustrated in Figure 1a. For a PPLN case, the pump beam propagates along the $y$-direction perpendicular to the ferroelectric domain wall, as shown in Figure 1b. Under the first-order QPM, an $x$-polarized pump photon with a frequency $2 \omega$ produces a pair of signal-idler photons with the same frequency $\omega$, and the pair has $x$ - and $z$-polarization states (or vice versa), respectively. The arrow in each 
ferroelectric domain represents the direction of spontaneous polarization $\left(P_{S}\right)$, and $\Lambda$ is the poling period for the first-order QPM.

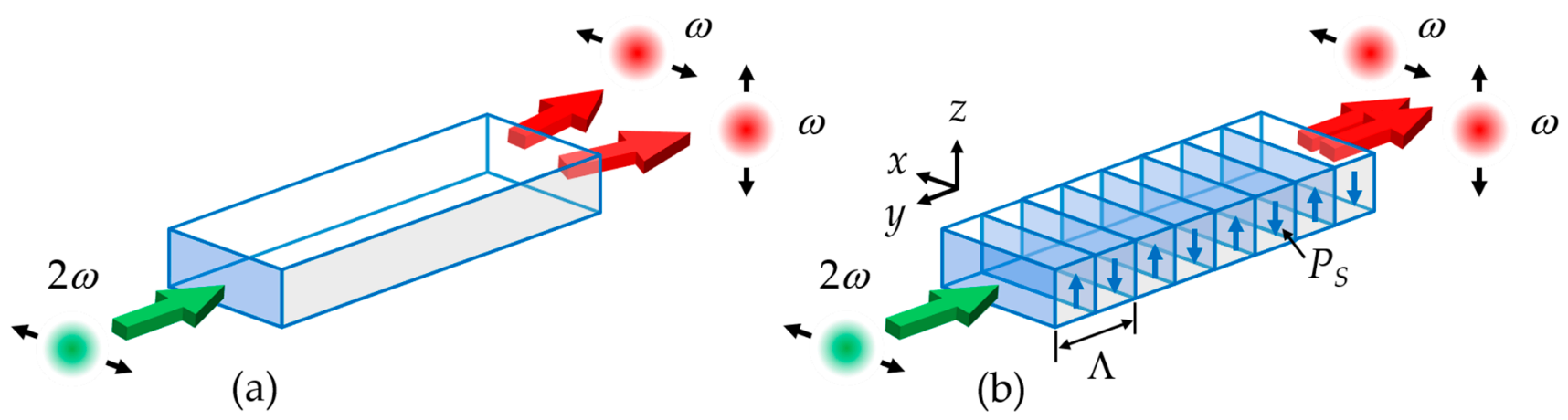

Figure 1. Schematic diagrams showing the frequency and polarization relationships of pump, signal, and idler photons interacting via Type II spontaneous parametric down-conversion (SPDC) in (a) a non-poled lithium niobate (LN) and (b) a periodically poled LN (PPLN).

For a non-poled LN shown in Figure 1a, the Type II PM condition can be expressed as,

$$
\Delta k=\left|k_{e}(2 \omega, \theta)-k_{o}(\omega)-k_{e}(\omega, \theta)\right|=0,
$$

where each $k$ represents the wave number of the interacting wave, and is defined as $k_{o}(\omega)=(\omega / c) n_{o}(\omega)$ or $k_{e}(l \omega, \theta)=(l \omega / c) n_{e}(l \omega, \theta)$. Here, $l$ can be 1 or 2 , and $c$ denotes the speed of light in vacuum. For light propagating at the angle $\theta$ to the optic axis of the LN, the RI of extraordinary wave, $n_{e}(\theta)$, can be derived as follows, using the definition in [18]:

$$
n_{e}(l \omega, \theta)=\frac{n_{o}(l \omega) n_{e}(l \omega)}{\sqrt{n_{o}^{2}(l \omega) \sin ^{2} \theta+n_{e}^{2}(l \omega) \cos ^{2} \theta}} .
$$

The RIs $\left(n_{e}\right.$ and $\left.n_{o}\right)$ of two kinds of LNs considered in this work (i.e., undoped and 5 -mol\% MgO-doped LNs) are found in [39,40].

For the PPLN configuration shown in Figure $1 b$, each of the three interacting photons is polarized along one of the principal axes of the index ellipsoid, respectively. The first order QPM condition under the Type II interaction (when using the nonlinear optic coefficient $\left.d_{15}\right)$ is then expressed as,

$$
\Delta k_{Q}=\left|k_{o}(2 \omega)-k_{o}(\omega)-k_{e}(\omega)\right|-\frac{2 \pi}{\Lambda}=0,
$$

where $k_{0}(l \omega)=(l \omega / c) n_{0}(l \omega)$ and $k_{e}(\omega)=(\omega / c) n_{e}(\omega)$. Here, $l$ can be 1 or 2 .

The temporal walk-off between the interacting photons due to the difference in GV is expressed as the time delay $(\Delta T)$ per unit crystal length as follows:

$$
\frac{\Delta T}{L}=\frac{\Delta n_{g}}{c},
$$

where $L$ and $\Delta n_{g}$ represent the crystal length and the group index difference between interacting photons, respectively [41]. Then, when $\Delta n_{g}=0$ in Equation (4), GV matching is achieved, which can be simplified as:

$$
\begin{aligned}
2 n_{e}^{(g)}(2 \omega, \theta) & =n_{o}^{(g)}(\omega)+n_{e}^{(g)}(\omega, \theta), \\
2 n_{o}^{(g)}(2 \omega) & =n_{o}^{(g)}(\omega)+n_{e}^{(g)}(\omega),
\end{aligned}
$$

where Equations (5) and (6) correspond to the case of non-poled LN and PPLN, respectively. Each superscript $g$ in Equations (5) and (6) means the group index. Now Type II EPM 
for each case shown in Figure 1 is defined as when Equations (1) and (5) (for a nonpoled LN in Figure 1a), or Equations (3) and (6) (for a PPLN in Figure 1b) are satisfied simultaneously. For a non-poled LN, Equations (1) and (5) can both be expressed as two-variable functions for $\theta$ and the pump wavelength $\left(\lambda_{p}\right)$. Therefore, by solving the system of Equations (1) and (5), a solution set of $\lambda_{p}$ and $\theta$ that satisfies Type II EPM can be obtained, where pure biphoton-states are produced. For a PPLN, Equations (3) and (6) are both given as a function of $\lambda_{p}$. Therefore, once a $\lambda_{p}$ that satisfies the GV matching condition of Equation (6) is obtained, the poling period $(\Lambda)$ for the first QPM is determined by Equation (3). PP structures with specific periods (>a few $\mu \mathrm{m}$, typically) can be easily fabricated with current ferroelectric domain engineering techniques. Therefore, an SPDCbased photon-pair source that satisfies Type II EPM can be implemented in a PPLN.

In negative uniaxial crystals with the point symmetry $3 \mathrm{~m}$, such as LN, the effective nonlinear-optic coefficient for Type II PM in a non-poled LN is given by [42],

$$
d_{e f f}=d_{22} \cos ^{2}(\theta+\rho) \sin 3 \phi,
$$

where $\phi$ and $\theta$ are the azimuthal and polar angles in the spherical coordinate, respectively. The nonlinear optic coefficient, $d_{22}$, is known to be $2.1 \pm 0.21 \mathrm{pm} / \mathrm{V}$ at $1064 \mathrm{~nm}[43,44]$. For a negative uniaxial crystal with the point symmetry $3 \mathrm{~m}$, the spatial walk-off angle $(\rho)$ between the wave vector and the Poynting vector within the crystal can be simplified to:

$$
\tan \rho=\frac{\sin 2 \theta}{2}\left(\frac{1}{n_{e}^{2}(\omega)}-\frac{1}{n_{o}^{2}(\omega)}\right) n_{e}^{2}(\omega, \theta),
$$

where $n_{e}(\omega, \theta)$ is given in Equation (2). To derive this expression, we used the definition in [45]. The azimuth angle has no effect on the PM condition as shown in Equations (1) and (2), so the maximum $d_{\text {eff }}$ can be obtained by setting $\phi$ to $\pi / 6$. As can be appreciated from Equations (7) and (8), $d_{\text {eff }}$ and $\rho$ are both functions of $\lambda_{p}$ and $\theta$. Therefore, for a non-poled LN, $d_{\text {eff }}$ can be obtained by substituting the solution sets of $\lambda_{p}$ and $\theta$ that satisfy Type II EPM (i.e., Equations (1) and (5)). For a PPLN, since Type II PM uses $d_{15}, d_{e f f}$ is given as $(2 / \pi) d_{15}$ for the first-order QPM. The SPDC efficiency is proportional to the square of the $d_{\text {eff }}$ for a given direction of beam propagation [4]. The nonlinear optic coefficients $d_{15}$ of two kinds of LNs (i.e., undoped and 5 mol\% MgO-doped LNs) are found in $[43,44,46]$.

The spatial walk-off between the interacting beams does not occur in the QPM situation shown in Figure 1b. However, when the beams generally do not propagate along the crystallographic axis, as shown in Figure 1a, the spatial walk-off occurs due to the difference in direction of the wave vector and the Poynting vector within the crystal. For the Type II interaction in a negative uniaxial crystal defined in Equation (1), the relationships of Poynting vectors (s) of the interacting waves are given as illustrated in Figure $2 b$, while the PM is collinear as shown in Figure 2a. For the ordinary wave, the wave vector and the Poynting vector are parallel to each other (i.e., $\mathbf{k}_{o}(\omega) / / \mathbf{s}_{o}(\omega)$ ), but this is not the case for the extraordinary waves. $\rho_{\omega}$ and $\rho_{2 \omega}$ in Figure $2 \mathrm{~b}$ are the walk-off angles of the extraordinary waves with frequencies $\omega$ and $2 \omega$ (Equation (8)), respectively, and in this study the relationship of $\rho_{2 \omega}>\rho_{\omega}$ is valid. Then, for an SPDC in a non-poled LN, the largest walk-off angle $(w)$ between the Poynting vectors of the interacting waves is equal to $\rho_{2 \omega} \omega$. As expected in Equation (8), $w$ is also the two-variable function for $\lambda_{p}$ and $\theta$, which can be obtained by substituting the solution set of $\lambda_{p}$ and $\theta$ that satisfies Type II PM. Then the maximum deviation between the interacting beams after passing through the crystal of length $L$ can be expressed as:

$$
\Delta=L \tan w
$$




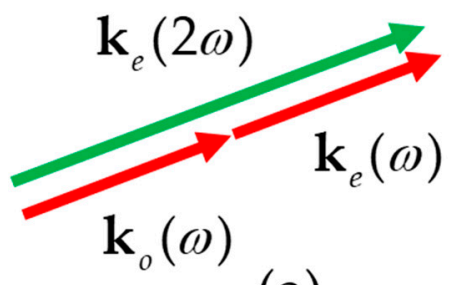

(a)

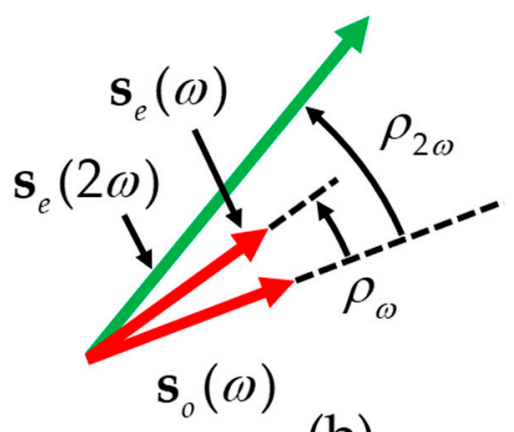

(b)

Figure 2. Example schematic diagrams showing the relationships of (a) wave vectors $\left(\mathbf{k}_{e}, \mathbf{k}_{o}\right)$ and (b) Poynting vectors ( $\mathbf{s}_{e}$, $\mathbf{s}_{o}$ ) of the interacting waves under Type II PM. Each $\rho$ represents the walk-off angle between $\mathbf{k}$ and $\mathbf{s}$. The angular scale is exaggerated.

The joint spectral analysis - the construction of the signal-idler joint spectral amplitude (JSA) and the calculation of the purity of the biphoton state via Schmidt decomposition-is commonly used to quantify the heralded-state spectral purity of the SPDC output [47-49]. Here, the spectral purity is a parameter that describes the degree of spectral uncorrelation between the signal and the idler photons. The signal-idler biphoton state $|\psi\rangle$ generated from SPDC can be expressed as,

$$
|\psi\rangle=\int_{0}^{\infty} \int_{0}^{\infty} d \omega_{s} d \omega_{i} f\left(\omega_{s}, \omega_{i}\right) \hat{a}_{s}^{\dagger}\left(\omega_{s}\right) \hat{a}_{i}^{\dagger}\left(\omega_{i}\right)|0\rangle|0\rangle,
$$

where $\hat{a}_{s}^{\dagger}$ and $\hat{a}_{i}^{\dagger}$ are the creation operators of the signal and idler photons, respectively, and $\omega_{s}$ and $\omega_{i}$ are the corresponding frequencies [49]. The normalized correlation function, $f\left(\omega_{s}, \omega_{i}\right)$, is expressed as the product of the pump envelope function and the PM function as follows:

$$
f\left(\omega_{s}, \omega_{i}\right)=\alpha\left(\omega_{s}, \omega_{i}\right) \varphi\left(\omega_{s}, \omega_{i}\right)
$$

which represents the biphoton JSA [47]. Assuming a pump with Gaussian spectral shape, the pump envelope (PE) function can be written as,

$$
\alpha\left(\omega_{s}, \omega_{i}\right) \propto \exp \left[-\frac{\left(\omega_{s}+\omega_{i}-\omega_{p}\right)^{2}}{\sigma_{p}^{2}}\right]
$$

where $\omega_{p}$ and $\sigma_{p}$ represent the center frequency and bandwidth of the pump, respectively. The PM function is given in the form of a sinc function as shown below,

$$
\varphi\left(\omega_{s}, \omega_{i}\right) \propto \operatorname{sinc}\left(\frac{\Delta k^{\prime} L}{2}\right),
$$

where $\Delta k^{\prime}$ represents the phase-mismatch defined in Equation (1) ( $\Delta k$ for a non-poled LN) or Equation (3) $\left(\Delta k_{Q}\right.$ for a PPLN). For the given $k_{p}$-direction (i.e., $\left.\theta\right)$ that satisfies the EPM, Equation (11) is given as a function of the signal and idler wavelengths (or $\lambda_{s}$ and $\left.\lambda_{i}\right)$, thus the JSA can be plotted on a two-dimensional plane as a function of $\lambda_{S}$ and $\lambda_{i}$. The purity can be calculated via the following Schmidt decomposition,

$$
f\left(\omega_{s}, \omega_{i}\right)=\sum_{j} \sqrt{c_{j}}\left|\zeta_{s, j}\right\rangle\left|\zeta_{i, j}\right\rangle
$$

where Schmidt coefficients, $c_{j}$, are a set of non-negative real numbers satisfying the normalization condition, $\sum_{j} c_{j}=1 .\left|\zeta_{s, j}\right\rangle$ and $\left|\zeta_{i, j}\right\rangle$ represent the orthonormal basis states called 
Schmidt modes [48]. Then the spectral purity, $P$, is defined as the sum of squares of Schmidt coefficients, as in [49]:

$$
P=\sum_{j} c_{j}^{2}
$$

Therefore, once we plot the JSA as functions of $\lambda_{s}$ and $\lambda_{i}$ in a two-dimensional plane, $P$ can be calculated via the Schmidt decomposition in Equation (15).

\section{Simulations and Discussion}

Figure 3 shows the numerical simulation results of Type II EPM in a PPLN (Figure 3a) and a 5-mol\% MgO-doped PPLN (MgO:PPLN) (Figure $3 \mathrm{~b}$ ) at temperature $t=50^{\circ} \mathrm{C} . \Lambda$ satisfying the first-order QPM (Equation (3)) and the temporal walk-off per unit crystal length $(\Delta T / L)$ defined in Equation (4) are plotted as a function of $\lambda_{p}$. The refractive indices used for the simulations are found in [39,40]. As indicated by the dashed circles and arrows in Figure 3, the $\lambda_{p}$ values for GV matching (i.e., $\Delta T / L=0$ ) are $1817.03 \mathrm{~nm}$ (for a PPLN) and $1757.35 \mathrm{~nm}$ (for a MgO:PPLN), and the corresponding $\Lambda$ s are $18.04 \mu \mathrm{m}$ and $18.35 \mu \mathrm{m}$, respectively. Examples of pump light sources at these wavelengths include Fabry-Pérot lasers, distributed feedback lasers, and super-luminescent diodes [50-52]. PP crystals with such long periods can be readily fabricated using current electric poling technology with the sub- $\mu \mathrm{m}$ resolution. Type II EPM properties calculated for two kinds of PPLNs are summarized in Table 1. The signal/idler wavelength in each case falls into the mid-IR range (i.e., $3634.06 \mathrm{~nm}$ and $3514.70 \mathrm{~nm}$ ). LN-based photon pair sources in this spectral range can be utilized in absorption spectroscopy to control the combustion and leakage of methane $\left(\mathrm{CH}_{4}\right)$ and to detect formaldehyde $\left(\mathrm{CH}_{2} \mathrm{O}\right)$ exposure [53,54]. In addition, the generation and detection of nonclassical light in the mid-IR range has good potential in an emerging field of high-sensitivity metrology, gravitational wave detection, and free-space quantum key distribution [35-38]. As indicated in Figure $3, \Lambda$ has an extremum at each $\lambda_{p}$ for GV matching because the derivative of $\Lambda$ by $\lambda_{p}$ is proportional to $\Delta n_{g}$ [41]. As described in Section 2, the Type II QPM uses the nonlinear optic coefficients $d_{15}$, and the values are listed in Table 1 together with references.
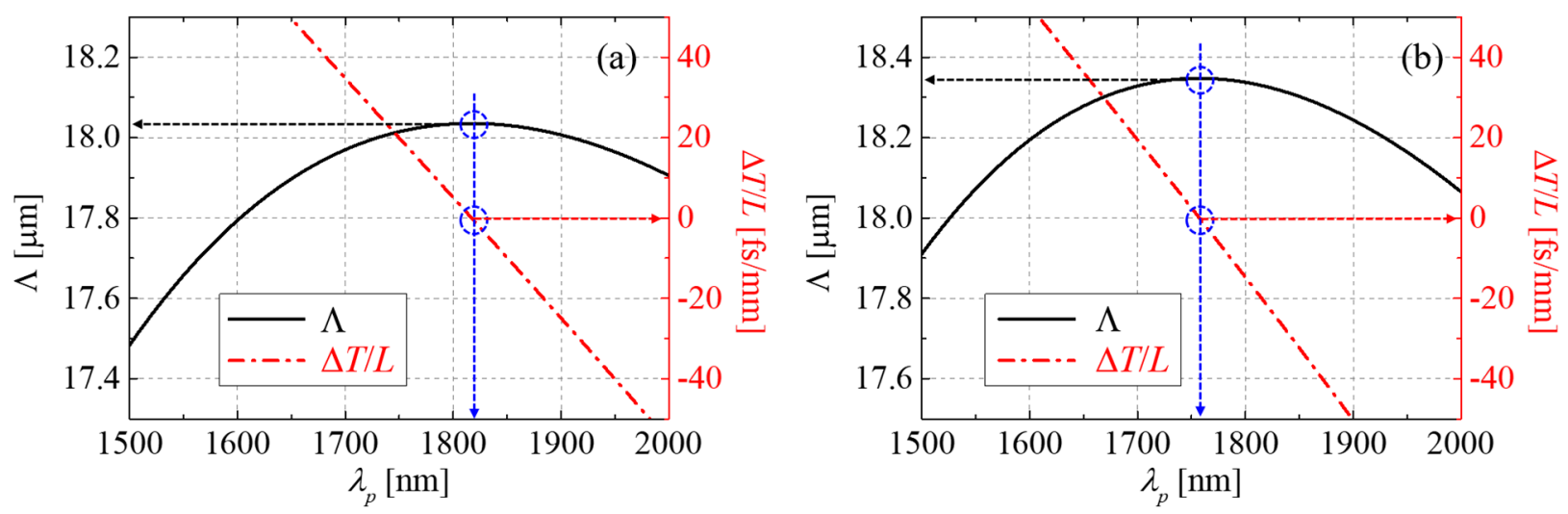

Figure 3. Numerical simulation results of Type II extended phase matching (EPM) in (a) a PPLN and (b) a 5-mol\% MgO:LN. The poling period $(\Lambda)$ satisfying the first-order quasi phase matching (QPM) and the temporal walk-off per unit crystal length $(\Delta T / L)$ are plotted as a function of $\lambda_{p}$.

Table 1. Type II EPM properties of two kinds of PPLNs: the poling periods $(\Lambda)$, the resonant pump wavelength $\left(\lambda_{p}\right)$, and the corresponding signal/idler wavelengths.

\begin{tabular}{ccccc}
\hline Crystal & $\left|d_{\mathbf{1 5}}\right|[\mathbf{p m} / \mathbf{V}]^{\mathbf{1}}$ & $\boldsymbol{\Lambda}[\mu \mathrm{m}]$ & $\lambda_{p}[\mathrm{~nm}]$ & $\lambda_{s, i}[\mathrm{~nm}]$ \\
\hline PPLN & $4.35 \pm 0.44$ & 18.04 & 1817.03 & 3634.06 \\
5-mol\% MgO:PPLN & {$[43,44]$} & 18.35 & 1757.35 & 3514.70 \\
\hline
\end{tabular}

${ }^{1}$ Assuming that Kleinman symmetry is valid, $d_{15}=d_{31}$. 
Figure 4 shows the numerical simulation results of Type II EPM in a non-poled LN (Figure 4a) and a non-poled MgO:LN (Figure $4 \mathrm{~b}$ ) at $t=50^{\circ} \mathrm{C}$. In each graph, the solid black and dash-dot red lines represent the PM and GV matching properties calculated using Equations (1) and (5), respectively. The intersection point of two curves represents the $k_{p}$ direction (i.e., $\theta$ ) and the corresponding $\lambda_{p}$ satisfying Type II EPM. The pump wavelengths for $\mathrm{GV}$ matching (i.e., $\Delta T / L=0$ ) are $1007.99 \mathrm{~nm}$ (for a $\mathrm{LN}$ ) and $1018.15 \mathrm{~nm}$ (for a MgO:LN), where the $k_{p}$-directions are $\theta=70.47^{\circ}$ and $\theta=74.58^{\circ}$, respectively. Type II EPM properties calculated for two non-poled LNs are summarized in Table 2 . The calculated wavelengths of photon pairs are $2015.99 \mathrm{~nm}$ and $2036.30 \mathrm{~nm}$, which are within the silica fiber transparency $(<2300 \mathrm{~nm})$ [55]. It is widely known that communication C-band is the best option for low-loss optical communication. However, it is necessary to use a wider bandwidth (e.g., $\mathrm{S}+\mathrm{C}+\mathrm{L}$ bands) to accommodate the increasing communication traffic [56,57]. Here, the Sand L-bands are used as many passive optical networks (PONs) and dense wavelength division multiplexing (DWDM) systems. Therefore, LN-based quantum light sources have good potential for demonstrating fiber-based broadband quantum communication. The generation and detection of nonclassical light about $2 \mu \mathrm{m}$ also has good potential, especially in gravitational wave detection [35].
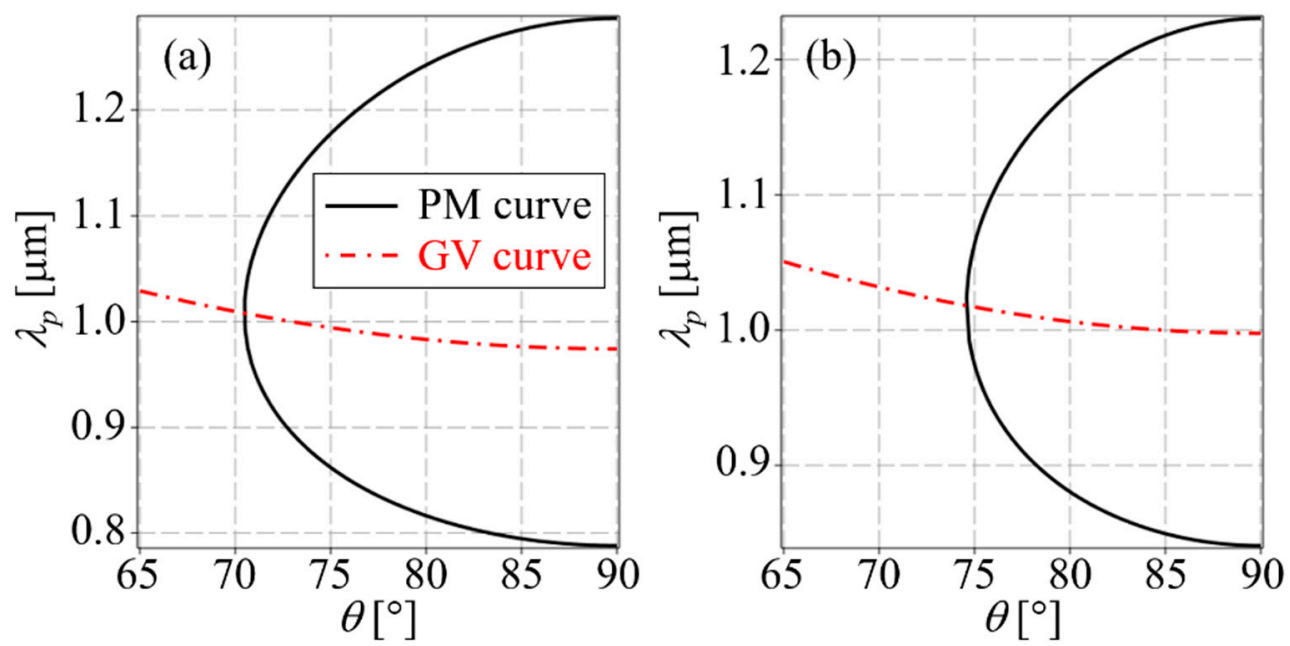

Figure 4. Numerical simulation results of Type II EPM in (a) a LN and (b) a 5-mol\% MgO:LN. In each graph, the intersection point of the PM and GV curves represent the $k_{p}$-direction (i.e., $\theta$ ) and the corresponding $\lambda_{p}$ satisfying Type II EPM.

Table 2. Type II EPM properties of two kinds of non-poled LNs: the PM direction $(\theta)$, the resonant pump wavelength $\left(\lambda_{p}\right)$ and the corresponding signal/idler wavelengths, $\left(\lambda_{s, i}\right)$, the effective nonlinear optic coefficients $\left(d_{\text {eff }}\right)$, the angle of walk-off between the interacting photons $(w)$, and the beam deviation $(\Delta)$.

\begin{tabular}{ccccccc}
\hline Crystal & $\boldsymbol{\theta}\left[^{\circ}\right]$ & $\lambda_{p}[\mathrm{~nm}]$ & $\lambda_{s, i}[\mathrm{~nm}]$ & $d_{e f f}[\mathrm{pm} / \mathrm{V}]$ & $w\left[^{\circ}\right]$ & $\Delta[\mu \mathrm{m} / \mathrm{mm}]$ \\
\hline LN & 70.47 & 1007.99 & 2015.99 & 0.21 & 1.30 & 22.73 \\
5-mol\% & 74.58 & 1018.15 & 2036.30 & 0.13 & 1.01 & 17.60 \\
MgO:LN & & & & & & \\
\hline
\end{tabular}

Since the SPDC efficiency is proportional to the square of $d_{\text {eff }}$, it is critical to estimate the effective nonlinearity for the given $k_{p}$-direction that satisfies Type II EPM. Figure 5 shows $d_{\text {eff }}$ and $\rho_{2 \omega}$ plotted as a function of $\lambda_{p}$, which are calculated numerically using Equations (7) and (8). The $\lambda_{p}$ values in the horizontal axes in Figure 5 correspond to the $\lambda_{p}$ range satisfying Type II PM shown in Figure 4 . Here, $\lambda_{p}$ is the solution to Equation (1), which is a solution pair with the PM direction, $\theta$. The $d_{\text {eff }}$ values calculated for Type II EPM are $0.21 \mathrm{pm} / \mathrm{V}$ for a LN and $0.13 \mathrm{pm} / \mathrm{V}$ for a MgO:LN as shown in Figure $5 \mathrm{a}$. Over the entire 
PM wavelength range, $d_{e f f}$ is larger for LN than for MgO:LN. In the PPLN configuration shown in Figure $1 b, d_{\text {eff }}$ is simply given as $(2 / \pi) d_{15}$ for the first-order QPM. Figure $5 b$ shows the walk-off angle $\rho_{2 \omega}$, calculated for the given PM solution set of $\lambda_{p}$ and $\theta$. Overall, also in the case of $\mathrm{LN}$, the walk-off is larger than that of $\mathrm{MgO}: \mathrm{LN}$. The calculated value of $\rho_{2 \omega}$ is used to calculate $d_{e f f}$ with Equation (8), and it also directly means the maximum walk-off angle $w$ between the interacting photons, as discussed in Section 2 . The $w$ values are calculated as $1.30^{\circ}$ for a $\mathrm{LN}$ and $1.01^{\circ}$ for a $\mathrm{MgO}: \mathrm{LN}$, which correspond to the beam deviation $(\Delta) 22.73 \mu \mathrm{m} / \mathrm{mm}$ and $17.60 \mu \mathrm{m} / \mathrm{mm}$, respectively. Here, each $\Delta$ was calculated using Equation (9), which can be sufficiently overcome by a large beam window in thick crystals. The calculated values of $d_{\text {eff }}, w$, and $\Delta$ are also summarized in Table 2.
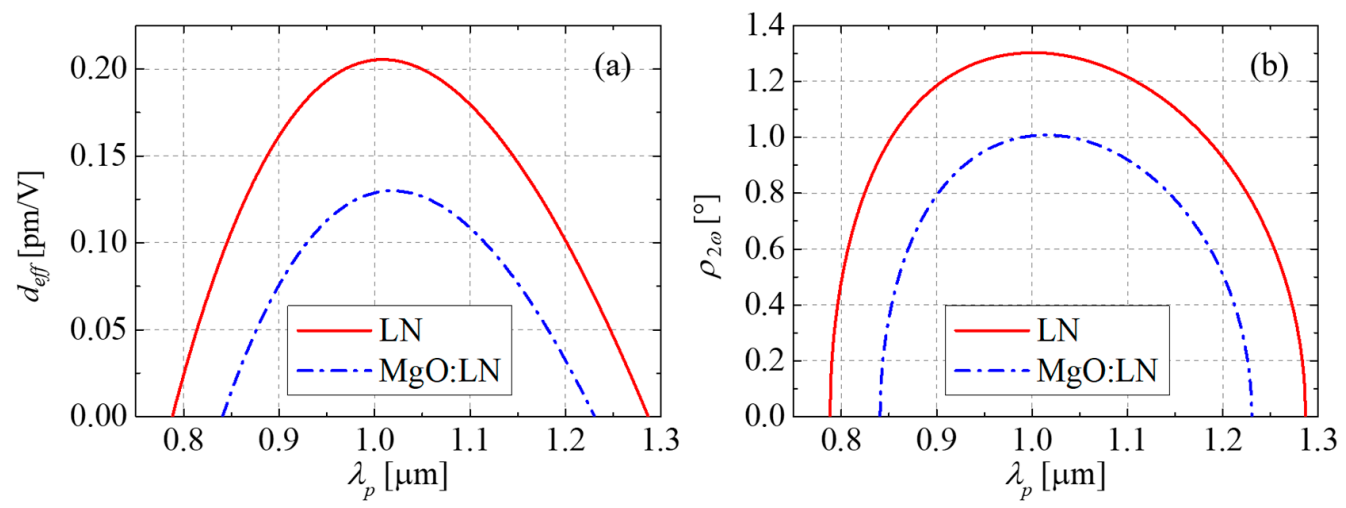

Figure 5. (a) Effective nonlinear optic coefficients $\left(d_{e f f}\right)$ under Type II EPM, numerically calculated for the two kinds of non-poled LNs: LN and 5-mol\% MgO:LN; (b) the walk-off angle $\rho_{2 \omega}$, calculated for the PM solution set of $\lambda_{p}$ and $\theta$.

Now we investigate the temperature behaviors of the Type II EPM properties of LNs. Figure 6 shows the change in $\lambda_{p}$ for Type II EPM (corresponding to the intersection points in Figure 4) and the PM direction $\theta$ at that point, which are plotted as a function of $t$ in two kinds of non-poled LNs. Here, $\Delta \lambda_{p}$ means the difference in $\lambda_{p}$ between the temperature $t$ and $t=50^{\circ} \mathrm{C}$ (i.e., $\left.\Delta \lambda_{p} \equiv \lambda_{p}(t)-\lambda_{p}\left(50^{\circ} \mathrm{C}\right)\right)$. As the temperature increases, $\lambda_{p}$ shifts to longer wavelengths $\left(\Delta \lambda_{p}>0\right)$ for LN (Figure 6a) and shorter wavelengths $\left(\Delta \lambda_{p}<0\right)$ for $5-\mathrm{mol} \% \mathrm{MgO}: \mathrm{LN}$ (Figure $6 \mathrm{~b}$ ). In the case of $\mathrm{LN}, \Delta \lambda_{p}$ is only $1.87 \mathrm{~nm}$ (corresponding to $3.74 \mathrm{~nm}$ spectral change in $\left.\Delta \lambda_{s, i}\right)$ for large temperature changes from $50^{\circ} \mathrm{C}$ to $150{ }^{\circ} \mathrm{C}$, which is sufficiently small compared to the spectral bandwidth of the photon pair $(\sim 7.6 \mathrm{~nm})$ to be described in a later paragraph. Since the spectral change for a temperature change of $100{ }^{\circ} \mathrm{C}$ is within the signal/idler spectral bandwidth, the LN-based photon-pair source operates stably against temperature change. For 5-mol\% MgO:LN, the Type II EPM point does not exist at temperatures above $80^{\circ} \mathrm{C}$ as plotted in Figure $6 \mathrm{~b} . \Delta \lambda_{p}$ for temperature changes from $50{ }^{\circ} \mathrm{C}$ to $80^{\circ} \mathrm{C}$ is $-0.16 \mathrm{~nm}$ (corresponding to a $0.32 \mathrm{~nm}$ spectral change in $\left.\Delta \lambda_{s, i}\right)$, which is also much smaller than the spectral bandwidth of the photon pair $(\sim 7.8 \mathrm{~nm})$ to be described in a later paragraph. Therefore, both $\mathrm{LN}$ and 5-mol\% MgO:LN can be suitable platforms for quantum light sources that are insensitive to temperature changes.

Figure 7a,b shows the temperature dependence of the first-order QPM (Equation (3)) and the temporal walk-off (Equation (4)) between the interacting photons. The solid black line in each graph represents the change in $\lambda_{p}$ as a function of $t$ of a PP crystal with the fixed poling period designed for Type II EPM at $t=50{ }^{\circ} \mathrm{C}\left(\equiv \Lambda_{50}{ }^{\circ} \mathrm{C}\right)$. The dash-dot red line in each graph represents the change in $\lambda_{p}$ for no temporal walk-off as a function of $t$. In both cases of PPLN (Figure 7a) and $5 \mathrm{~mol} \%$-MgO:PPLN (Figure $7 \mathrm{~b}$ ), $\lambda_{p}$ for the first-order QPM appears even when the temperature of PP crystals change. However, the GV matching is not achieved except for $t=50^{\circ} \mathrm{C}$. Figure 7c shows the PM function (Equation (13)) plotted as a function of $t$, which means the spectral responses of the PP crystals to temperature. The bandwidths (full-width-half-maximum) are calculated as $>52{ }^{\circ} \mathrm{C}$ for PPLN and $1.90{ }^{\circ} \mathrm{C}$ for $5 \mathrm{~mol} \%-\mathrm{MgO}: P P L N$. Therefore, in application as a quantum light source, PPLN is not 
sensitive to temperature changes, while $5 \mathrm{~mol} \%-\mathrm{MgO}: \mathrm{PPLN}$ needs to be maintained at an appropriate temperature.
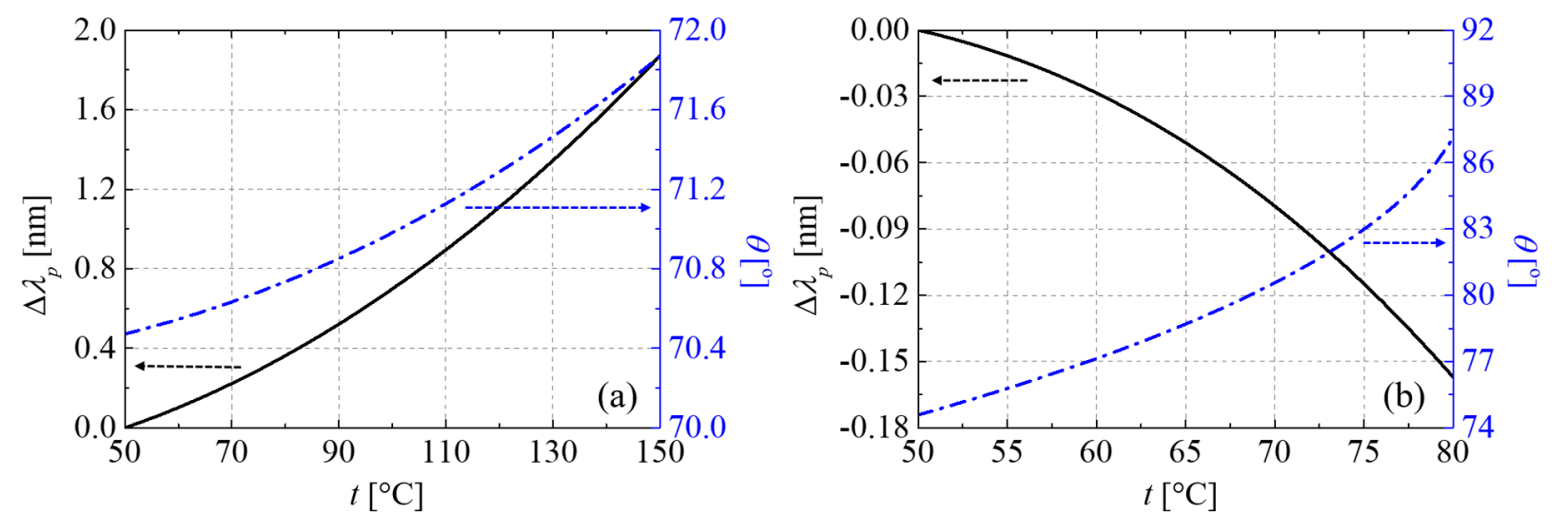

Figure 6. Temperature behaviors of the resonant $\lambda_{p}$ and the corresponding PM direction $(\theta)$ in two kinds of non-poled LNs: (a) LN and (b) 5 mol\%-MgO:LN.
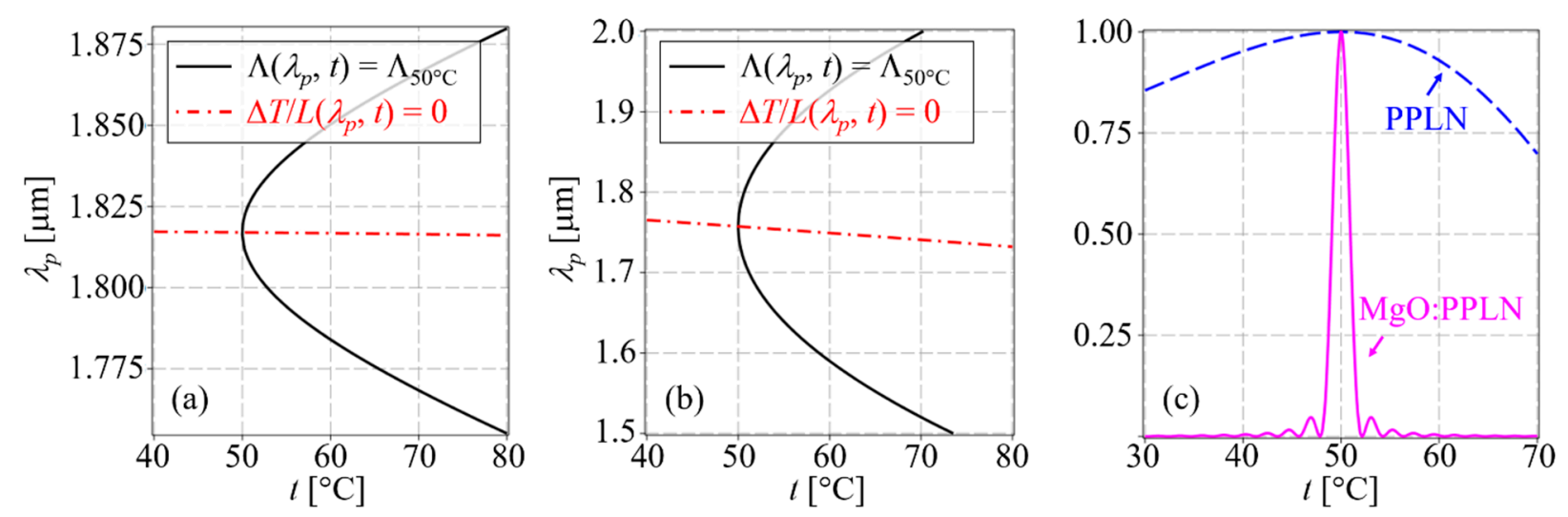

Figure 7. Temperature behaviors of the resonant $\lambda_{p}$ for the first order QPM and the temporal walk-offs in (a) PPLN and (b) $5 \mathrm{~mol} \%-\mathrm{MgO}: \mathrm{PPLN}$; (c) the spectral responses of the PP crystals to temperature.

In order to quantify the heralded-state spectral purity of SPDC output, we now discuss the analysis of the signal-idler JSA properties including the calculation of the purity of the biphoton state. Figure 8 shows the joint spectral properties of Type II SPDC in the cases listed in Tables 1 and 2. The density plots of the JSAs (Equation (11)) for LN and $5 \mathrm{~mol} \%$ $\mathrm{MgO}: \mathrm{LN}$ are shown in Figure 8a,b, respectively. JSAs of PPLN and $5 \mathrm{~mol} \% \mathrm{MgO}: \mathrm{PPLN}$ are plotted in Figure 8c,d, respectively. The crystal length used for the calculations is $10 \mathrm{~mm}$ for all four cases. The bandwidths of PE functions (Equation (12)) chosen for higher purities are $2.27 \mathrm{~nm}, 2.33 \mathrm{~nm}, 5.33 \mathrm{~nm}$, and $5.17 \mathrm{~nm}$, respectively, in the order of Figure 8a-d. As can be seen in Figure 8, the JSAs show typical circular shapes as in the case of PPKTP under Type II EPM [24]. The contour lines for the joint spectral intensity (JSI) $=0.5$ are plotted in Figure 9, where Figure $9 a-d$, in turn, correspond to Figure $8 a-d$. Here, the JSI is defined as $|J S A|^{2}$. For clear comparison of the spectral bandwidths of the photon pairs, all contour plots in Figure 9 are displayed in a $20 \mathrm{~nm} \times 20 \mathrm{~nm}$ window. The contour lines show almost perfect circular shapes with the diameters of $7.6 \mathrm{~nm}, 7.8 \mathrm{~nm}, 17.3 \mathrm{~nm}$, and $17.8 \mathrm{~nm}$, in the order of Figure $9 \mathrm{a}-\mathrm{d}$. The purities calculated using Schmidt decomposition (Equation (15)) are as high as $0.995,0.995,0.999$, and 0.998, respectively, in the order of Figure 8a-d. The results show that very high-purity photon pairs can be generated in both PP and non-poled LNs. 

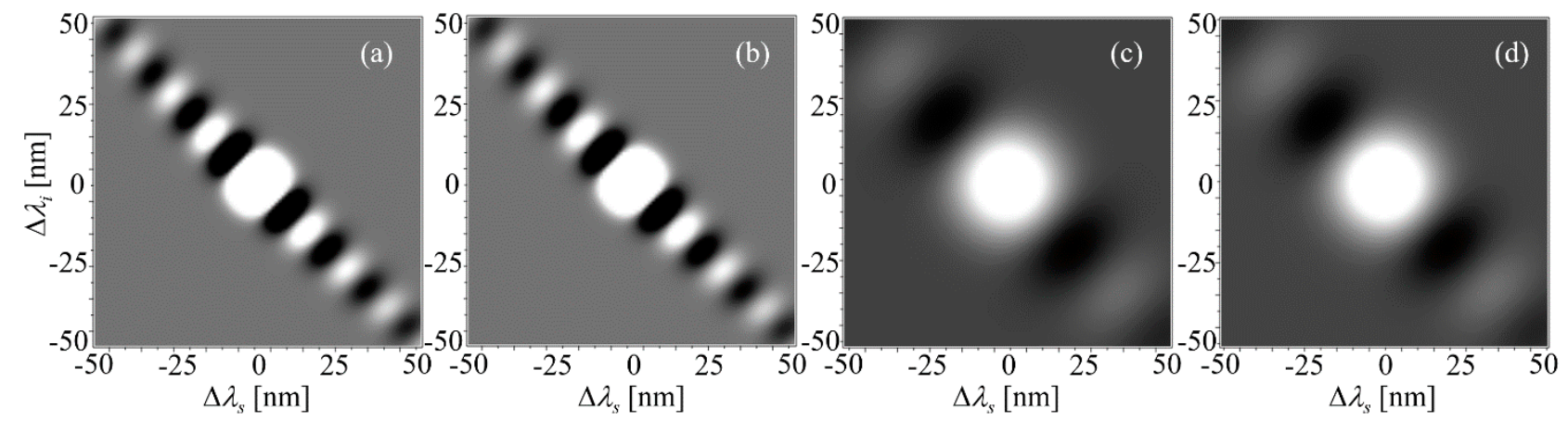

Figure 8. Joint spectral amplitudes (JSAs) of Type II SPDC in (a) LN, (b) 5 mol\%-MgO:LN (c) PPLN, and (d) 5 mol\%MgO:PPLN. The calculated purities are 0.995, 0.995, 0.999, and 0.998, respectively.
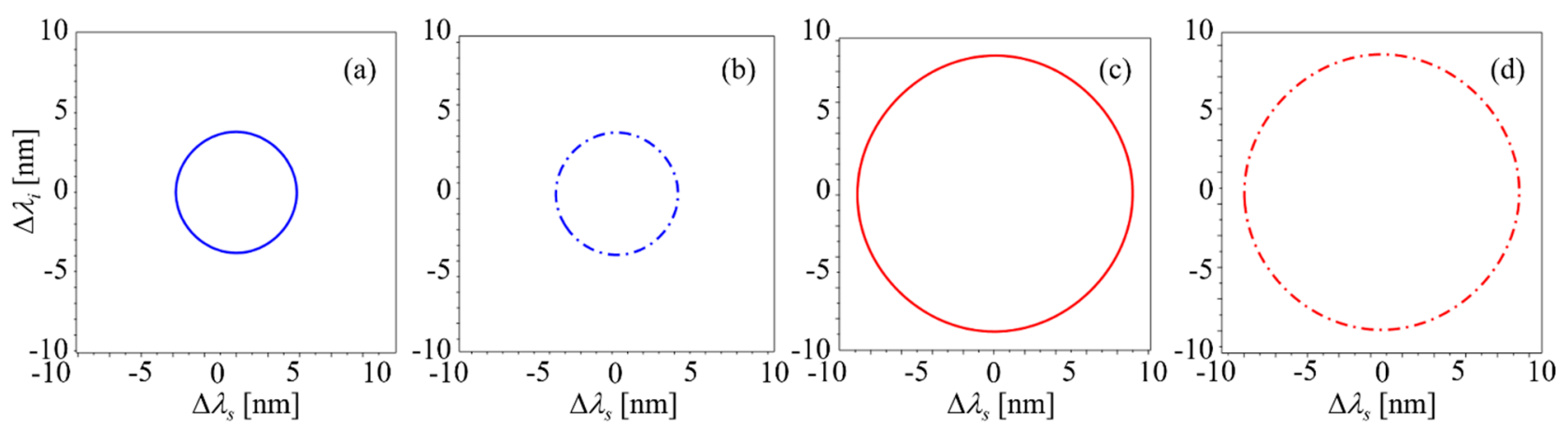

Figure 9. Contour lines for joint spectral intensity (JSI) $=0.5$ for the cases shown in Figure 8: (a) LN, (b) $5 \mathrm{~mol} \%-\mathrm{MgO}: \mathrm{LN}$ (c) PPLN, and (d) 5 mol\%-MgO:PPLN. The diameters of the circles are $7.6 \mathrm{~nm}, 7.8 \mathrm{~nm}, 17.3 \mathrm{~nm}$, and $17.8 \mathrm{~nm}$, in the order of Figure $9 \mathrm{a}-\mathrm{d}$. Each result was calculated for a crystal length of $10 \mathrm{~mm}$.

\section{Conclusions}

We have theoretically and numerically investigated Type II SPDC properties of two kinds of LNs-undoped LN and 5-mol\% MgO:LN. In both non-poled and PP crystals, SPDC properties under Type II EPM were theoretically investigated, in terms of PM, GV matchings, effective nonlinearities, and spatial walk-offs between the interacting photons. The spectral position of photon pairs can be chosen in the mid-IR region including $2015.99 \mathrm{~nm}, 2036.30 \mathrm{~nm}, 3634.06 \mathrm{~nm}$, and $3514.70 \mathrm{~nm}$, which has high potential for free-space quantum communication, spectroscopy, and high-sensitivity metrology. The heralded-state spectral purities of SPDC output were quantified via JSA analyses, and the results show that the purities calculated with proper pump filtering are as high as 0.995-0.999. While the acceptable temperature bandwidth of a MgO:PPLN-based SPDC source operating with a stable spectrum is less than $1.9^{\circ} \mathrm{C}$, other counterparts based on PPLN or two kinds of non-poled LNs are not sensitive to temperature changes. Nevertheless, practically all four cases do not require temperature precision equipment, which is a huge advantage for practical use of LN-based quantum light sources outside the laboratory. LN-based quantum light sources are expected to provide a variety of functions for SPDCbased quantum communication, information processing, metrology, and spectroscopy.

Author Contributions: Conceptualization, K.J.L.; Simulation, D.L.; writing-original draft preparation, I.K.; writing-review and editing, K.J.L.; supervision, K.J.L. All authors have read and agreed to the published version of the manuscript.

Funding: This research was funded by the National Research Foundation of Korea (Grant NRF2019R1F1A1063937); Korea Institute of Science and Technology (Grant 2E29580-19-147); and an Institute for Information \& Communications Technology Promotion (IITP) grant funded by the Korea government (MSIT) (No. 2020-0-00947). 
Institutional Review Board Statement: Not applicable.

Informed Consent Statement: Not applicable.

Data Availability Statement: The data presented in this study are available in this article.

Conflicts of Interest: The authors declare no conflict of interest.

\section{References}

1. Volk, T.; Wöhlecke, M. Lithium Niobate; Springer: Berlin, Germany, 2008; pp. 1-7.

2. Jia, Y.; Wang, L.; Chen, F. Ion-cut lithium niobate on insulator technology: Recent advances and perspectives featured. Appl. Phys. Rev. 2021, 8, 011307. [CrossRef]

3. Poberaj, G.; Hu, H.; Sohler, W.; Günter, P. Lithium niobate on insulator (LNOI) for micro-photonic devices. Laser Photon. Rev. 2012, 6, 488-503. [CrossRef]

4. Jinan Jingzheng Electronics Co.,Ltd. (nanoln). Available online: https:/ / www.nanoln.com (accessed on 25 February 2021).

5. Wang, C.; Zhang, M.; Chen, X.; Bertrand, M.; Shams-Ansari, A.; Chandrasekhar, S.; Winzer, P.; Lončar, M. Integrated lithium niobate electro-optic modulators operating at CMOS-compatible voltages. Nature 2018, 562, 101-104. [CrossRef] [PubMed]

6. Wang, C.; Langrock, C.; Marandi, A.; Jankowski, M.; Zhang, M.; Desiatov, B.; Fejer, M.M.; Lončar, M. Ultrahigh-efficiency wavelength conversion in nanophotonic periodically poled lithium niobate waveguides. Optica 2018, 5, 1438-1441. [CrossRef]

7. Wang, C.; Zhang, M.; Yu, M.; Zhu, R.; Hu, H.; Lončar, M. Monolithic lithium niobate photonic circuits for Kerr frequency comb generation and modulation. Nat. Commun. 2019, 10, 978. [CrossRef]

8. Yu, M.; Wang, C.; Zhang, M.; Lončar, M. Chip-based lithium-niobate frequency combs. IEEE Photon. Technol. Lett. 2019, 31, 1894-1897. [CrossRef]

9. Yu, M.; Desiatov, B.; Okawachi, Y.; Gaeta, A.L.; Lončar, M. Coherent two-octave spanning supercontinuum generation in lithium-niobate waveguides. Opt. Lett. 2019, 44, 1222-1225. [CrossRef]

10. Okawachi, Y.; Yu, M.; Desiatov, B.; Kim, B.Y.; Hansson, T.; Lončar, M.; Gaeta, A.L. Chip-based self-referencing using integrated lithium niobate waveguides. Optica 2020, 7, 702-707. [CrossRef]

11. Pohl, D.; Reig Escalé, M.; Madi, M.; Kaufmann, F.; Brotzer, P.; Sergeyev, A.; Guldimann, B.; Giaccari, P.; Alberti, E.; Meier, U.; et al. An integrated broadband spectrometer on thin-film lithium niobate. Nat. Photonics 2020, 14, 24-29. [CrossRef]

12. Xu, M.; He, M.; Zhang, H.; Jian, J.; Pan, Y.; Liu, X.; Chen, L.; Meng, X.; Chen, H.; Li, Z.; et al. High-performance coherent optical modulators based on thin-film lithium niobate platform. Nat. Commun. 2020, 11, 3911. [CrossRef]

13. Desiatov, B.; Shams-Ansari, A.; Zhang, M.; Wang, C.; Lončar, M. Ultra-low loss integrated visible photonics using thin-film lithium niobite. Optica 2019, 6, 380-384. [CrossRef]

14. Holzgrafe, J.; Sinclair, N.; Zhu, D.; Shams-Ansari, A.; Colangelo, M.; Hu, Y.; Zhang, M.; Berggren, K.K.; Lončar, M. Cavity electro-optics in thin-film lithium niobate for efficient microwave-to-optical transduction. Optica 2020, 7, 1714-1720. [CrossRef]

15. Luke, K.; Kharel, P.; Reimer, C.; He, L.; Loncar, M.; Zhang, M. Wafer-scale low-loss lithium niobate photonic integrated circuits. Opt. Express 2020, 28, 24452-24458. [CrossRef] [PubMed]

16. Shao, L.; Sinclair, N.; Leatham, J.; Hu, Y.; Yu, M.; Turpin, T.; Crowe, D.; Lončar, M. Integrated microwave acousto-optic frequency shifter on thin-film lithium niobate. Opt. Express 2020, 28, 23728-23738. [CrossRef]

17. Jankowski, M.; Langrock, C.; Desiatov, B.; Marandi, A.; Wang, C.; Zhang, M.; Phillips, C.R.; Lončar, M.; Fejer, M.M. Ultrabroadband nonlinear optics in nanophotonic periodically poled lithium niobate waveguides. Optica 2020, 7, 40-46. [CrossRef]

18. Boyd, R.W. Nonlinear Optics, 4th ed.; Academic Press: San Diego, CA, USA, 2020; pp. 77-79.

19. Kwiat, P.; Mattle, K.; Weinfurter, H.; Zeilinger, A.; Sergienko, A.V.; Shih, Y. New high-intensity source of polarization-entangled photon pairs. Phys. Rev. Lett. 1995, 75, 4337-4341. [CrossRef] [PubMed]

20. Steinlechner, F.; Gilaberte, M.; Jofre, M.; Scheidl, T.; Torres, J.P.; Pruneri, V.; Ursin, R. Efficient heralding of polarization-entangled photons from type-0 and type-II spontaneous parametric downconversion in periodically poled $\mathrm{KTiOPO}_{4}$. J. Opt. Soc. Am. B 2014, 31, 2068-2076. [CrossRef]

21. Giovannetti, V.; Maccone, L.; Shapiro, J.H.; Wong, F.N.C. Extended phase-matching conditions for improved entanglement generation. Phys. Rev. A 2002, 66, 043813. [CrossRef]

22. Jin, R.-B.; Shimizu, R.; Wakui, K.; Fujiwara, M.; Yamashita, T.; Miki, S.; Terai, H.; Wang, Z.; Sasaki, M. Pulsed Sagnac polarizationentangled photon source with a PPKTP crystal at telecom wavelength. Opt. Express 2014, 22, 11498-11507. [CrossRef]

23. Li, Y.; Zhou, Z.-Y.; Ding, D.-S.; Shi, B.-S. CW-pumped telecom band polarization entangled photon pair generation in a Sagnac interferometer. Opt. Express 2015, 23, 28792-28800. [CrossRef]

24. Weston, M.M.; Chrzanowski, H.M.; Wollmann, S.; Boston, A.; Ho, J.; Shalm, L.K.; Verma, V.B.; Allman, M.S.; Nam, S.W.; Patel, R.B.; et al. Efficient and pure femtosecond-pulse-length source of polarization-entangled photons. Opt. Express 2016, 24, 10869-10879. [CrossRef] [PubMed]

25. Kim, I.; Lee, D.; Lee, K.J. Numerical investigation of high-purity polarization-entangled photon-pair generation in non-poled KTP Isomorphs. Appl. Sci. 2021, 11, 565. [CrossRef]

26. Fujii, G.; Namekata, N.; Motoya, M.; Kurimura, S.; Inoue, S. Bright narrowband source of photon pairs at optical telecommunication wavelengths using a type-II periodically poled lithium niobate waveguide. Opt. Express 2007, 15, 12769-12776. [CrossRef] [PubMed] 
27. Martin, A.; Issautier, A.; Herrmann, H.; Sohler, W.; Ostrowsky, D.B.; Alibart, O.; Tanzilli, S. A polarization entangled photon-pair source based on a type-II PPLN waveguide emitting at a telecom wavelength. New. J. Phys. 2010, 12, 103005. [CrossRef]

28. Cheng, X.; Sarihan, M.C.; Chang, K.-C.; Lee, Y.S.; Laudenbach, F.; Ye, H.; Yu, Z.; Wong, C.W. Design of spontaneous parametric down-conversion in integrated hybrid $\mathrm{Si}_{x} \mathrm{~N}_{\mathrm{y}}$-PPLN waveguides. Opt. Express 2019, 27, 30773-30787. [CrossRef]

29. Kuo, P.S.; Verma, V.B.; Nam, S.W. Demonstration of a polarization-entangled photon-pair source based on phase-modulated PPLN. OSA Continuum 2020, 3, 295-304. [CrossRef]

30. Kuo, P.S.; Gerrits, T.; Verma, V.; Nam, S.W.; Slattery, O.; Ma, L.; Tang, X. Characterization of type-II spontaneous parametric down-conversion in domain-engineered PPLN. In Proceedings of the SPIE Advances in Photonics of Quantum Computing Memory, and Communication IX, San Francisco, CA, USA, 13-18 February 2016; SPIE: Bellingham, WA, USA, 2016.

31. Zhao, J.; Ma, C.; Rüsing, M.; Mookherjea, S. High quality entangled photon pair generation in periodically poled thin-film lithium niobate waveguides. Phys. Rev. Lett. 2020, 124, 163603. [CrossRef]

32. Chen, J.-Y.; Sua, Y.M.; Ma, Z.-H.; Tang, C.; Li, Z.; Huang, Y.-P. Efficient parametric frequency conversion in lithium niobate nanophotonic chips. OSA Continuum 2019, 2, 2914-2924. [CrossRef]

33. Schlarb, U.; Betzler, K. 'Influence of the defect structure on the refractive indices of undoped and Mg-doped lithium niobate. Phys. Rev. B 1994, 50, 751. [CrossRef]

34. Zelmon, D.E.; Small, D.L.; Jundt, D. Infrared corrected Sellmeier coefficients for congruently grown lithium niobate and 5 mol. \% magnesium oxide-doped lithium niobate. J. Opt. Soc. Am. B 1997, 14, 3319-3322. [CrossRef]

35. Prabhakar, S.; Shields, T.; Dada, A.C.; Ebrahim, M.; Taylor, G.G.; Morozov, D.; Erotokritou, K.; Miki, S.; Yabuno, M.; Terai, H.; et al Two-photon quantum interference and entanglement at $2.1 \mu \mathrm{m}$. Sci. Adv. 2020, 6, eaay5195. [CrossRef]

36. Rosenfeld, L.M.; Sulway, D.A.; Sinclair, G.F.; Anant, V.; Thompson, M.G.; Rarity, J.G.; Silverstone, J.W. Mid-infrared quantum optics in silicon. Opt. Express 2020, 28, 37092-37102. [CrossRef] [PubMed]

37. Mancinelli, M.; Trenti, A.; Piccione, S.; Fontana, G.; Dam, J.S.; Tidemand-Lichtenberg, P.; Pedersen, C.; Pavesi, L. Mid-infrared coincidence measurements on twin photons at room temperature. Nat. Commun. 2017, 8, 15184. [CrossRef]

38. Sua, Y.M.; Fan, H.; Shahverdi, A.; Chen, J.Y.; Huang, Y.P. Direct generation and detection of quantum correlated photons with 3.2 $\mu \mathrm{m}$ wavelength spacing. Sci. Rep. 2017, 7, 17494. [CrossRef]

39. Edwards, G.J.; Lawrence, M. A temperature-dependent dispersion equation for congruently grown lithium niobate. Opt. Quant. Electron. 1984, 16, 373-375. [CrossRef]

40. Gayer, O.; Sacks, Z.; Galun, E.; Arie, A. Temperature and wavelength dependent refractive index equations for MgO-doped congruent and stoichiometric $\mathrm{LiNbO}_{3}$. Appl. Phys. B 2008, 91, 343-348. [CrossRef]

41. Lee, K.J.; Lee, S.; Shin, H. Extended phase matching properties of periodically poled potassium niobate crystals for mid-infrared polarization-entangled photon-pair generation. Appl. Opt. 2016, 55, 9791-9796. [CrossRef] [PubMed]

42. Shoji, I.; Nakamura, H.; Ohdaira, K.; Kondo, T.; Ito, R.; Okamoto, T.; Tatsuki, K.; Kubota, S. Absolute measurement of second-order nonlinear-optical coefficients of $\beta-\mathrm{BaB}_{2} \mathrm{O}_{4}$ for visible to ultraviolet second-harmonic wavelengths. J. Opt. Soc. Am. B 1999, 16, 620-624 [CrossRef]

43. Miller, R.C.; Nordland, W.A.; Bridenbaugh, P.M. Dependence of second-harmonic-generation coefficients of LiNbO 3 on melt composition. J. Appl. Phys. 1971, 42, 4145-4147. [CrossRef]

44. Roberts, D.A. Simplified characterization of uniaxial and biaxial nonlinear optical crystals: A plea for standardization of nomenclature and conventions. IEEE J. Quant. Electron. 1992, 28, 2057-2074. [CrossRef]

45. Gehr, R.J.; Kimmel, M.W.; Smith, A.V. Simultaneous spatial and temporal walk-off compensation in frequency-doubling femtosecond pulses in $\beta-\mathrm{BaB}_{2} \mathrm{O}_{4}$. Opt. Lett. 1998, 23, 1298-1300. [CrossRef]

46. Shoji, I.; Kondo, T.; Kitamoto, A.; Shirane, M.; Ito, R. Absolute scale of second-order nonlinear-optical coefficients. J. Opt. Soc. Am. $B$ 1997, 14, 2268-2294. [CrossRef]

47. Grice, W.P.; Walmsley, I.A. Spectral information and distinguishability in type-II down-conversion with a broadband pump. Phys. Rev. A 1997, 56, 1627. [CrossRef]

48. Law, C.K.; Walmsley, I.A.; Eberly, J.H. Continuous frequency entanglement: Effective finite Hilbert space and entropy control. Phys. Rev. Lett. 2000, 84, 5304. [CrossRef] [PubMed]

49. Mosley, P.J.; Lundeen, J.S.; Smith, B.J.; Walmsley, I.A. Conditional preparation of single photons using parametric downconversion: A recipe for purity. New. J. Phys. 2008, 10, 093011. [CrossRef]

50. Nanoplus GmbH. Available online: https://nanoplus.com/en/products/fabry-perot-lasers-fp/1700-nm-2400-nm (accessed on 31 March 2021).

51. Nanoplus GmbH. Available online: https://nanoplus.com/en/products/distributed-feedback-lasers-dfb/1650-nm-1850-nm (accessed on 31 March 2021).

52. Nanoplus GmbH. Available online: https://nanoplus.com/en/products/superluminescent-diodes-sld/1700-nm-2300-nm (accessed on 31 March 2021).

53. Nanoplus GmbH. Available online: https://nanoplus.com/en/applications/applications-by-gas (accessed on 15 February 2021).

54. Whittaker, R.; Erven, C.; Neville, A.; Berry, M.; O’Brien, J.L.; Cable, H.; Matthews, J.C.F. Absorption spectroscopy at the ultimate quantum limit from single-photon states. New J. Phys. 2017, 19, 023013. [CrossRef]

55. Thorlabs Inc. Available online: https://www.thorlabs.com/thorproduct.cfm?partnumber=SM2000 (accessed on 25 February 2021). 
56. Iqbal, M.A.; Krzeczunowicz, L.; Phillips, I.; Harper, P.; Forysiak, W. Evaluation of performance penalty from pump-signal overlap in $\mathrm{S}+\mathrm{C}+\mathrm{L}$ band discrete Raman amplifiers. In Proceedings of the Optical Fiber Communication Conference (OFC), San Diego, CA, USA, 8-12 March 2020.

57. Hamaoka, F.; Nakamura, M.; Okamoto, S.; Minoguchi, K.; Sasai, T.; Matsushita, A.; Yamazaki, E.; Kisaka, Y. Ultra-wideband WDM transmission in S-, C-, and L-bands using signal power optimization scheme. J. Lightwave Technol. 2019, 37, 1764. [CrossRef] 